\title{
Knee adduction moments are not increased in obese knee osteoarthritis patients during stair negotiation
}

Citation for published version (APA):

Verlaan, L., Boekesteijn, R. J., Oomen, P. W., Liu, W. Y., Peters, M. J. M., Emans, P. J., van Rhijn, L. W., \& Meijer, K. (2019). Knee adduction moments are not increased in obese knee osteoarthritis patients during stair negotiation. Gait \& Posture, 73, 154-160. https://doi.org/10.1016/j.gaitpost.2019.07.192

Document status and date:

Published: 01/09/2019

DOI:

10.1016/j.gaitpost.2019.07.192

Document Version:

Publisher's PDF, also known as Version of record

Document license:

Taverne

Please check the document version of this publication:

- A submitted manuscript is the version of the article upon submission and before peer-review. There can be important differences between the submitted version and the official published version of record.

People interested in the research are advised to contact the author for the final version of the publication, or visit the DOI to the publisher's website.

- The final author version and the galley proof are versions of the publication after peer review.

- The final published version features the final layout of the paper including the volume, issue and page numbers.

Link to publication

\footnotetext{
General rights Owners
rights.

- You may freely distribute the URL identifying the publication in the public portal. please follow below link for the End User Agreement:

www.umlib.nl/taverne-license

Take down policy

If you believe that this document breaches copyright please contact us at:

repository@maastrichtuniversity.nl

providing details and we will investigate your claim.
}

Copyright and moral rights for the publications made accessible in the public portal are retained by the authors and/or other copyright owners and it is a condition of accessing publications that users recognise and abide by the legal requirements associated with these

- Users may download and print one copy of any publication from the public portal for the purpose of private study or research.

- You may not further distribute the material or use it for any profit-making activity or commercial gain

If the publication is distributed under the terms of Article $25 \mathrm{fa}$ of the Dutch Copyright Act, indicated by the "Taverne" license above, 
Full length article

\title{
Knee adduction moments are not increased in obese knee osteoarthritis patients during stair negotiation
}

\author{
L. Verlaan ${ }^{\mathrm{a}, *}$, R.J. Boekesteijn ${ }^{\mathrm{b}}$, P.W. Oomen ${ }^{\mathrm{a}, \mathrm{b}}$, W.Y. Liu ${ }^{\mathrm{b}}$, M.J.M. Peters ${ }^{\mathrm{a}}$, P.J. Emans ${ }^{\mathrm{a}}$, \\ L.W. van Rhijn ${ }^{\mathrm{a}}$, K. Meijer ${ }^{\mathrm{b}}$ \\ a Department of Orthopaedic Surgery, CAPHRI School for Public Health and Primary Care, Maastricht University Medical Center, P.O. Box 5800, 6202 AZ, Maastricht, the \\ Netherlands \\ ${ }^{\mathrm{b}}$ Department of Nutrition and Movement Sciences, NUTRIM School of Nutrition and Translational Research in Metabolism, Maastricht University Medical Center, P.O. Box \\ 616, 6200 MD, Maastricht, the Netherlands
}

\section{A R T I C L E I N F O}

\section{Keywords:}

Obesity

Knee osteoarthritis

Stair negotiation

Knee adduction moment

Biomechanics

\begin{abstract}
A B S T R A C T
Background: Negotiating stairs is an important activity of daily living that is also associated with large loads on the knee joint. In medial compartment knee osteoarthritis, the knee adduction moment during level walking is considered a marker for disease severity. It could be argued that the discriminative capability of this parameter is even better if tested in a strenuous stair negotiation task.

Research question: What is the relation with knee osteoarthritis on the knee adduction moment during the stance phase of both stair ascent and descent in patients with and without obesity?

Methods: This case control study included 22 lean controls, 16 lean knee osteoarthritis patients, and 14 obese knee osteoarthritis patients. All subjects ascended and descended a two-step staircase at a self-selected, comfortable speed. Three-dimensional motion analysis was performed to evaluate the knee adduction moment during stair negotiation.

Results: Obese knee osteoarthritis patients show a prolonged stance time together with a more flattened knee adduction moment curve during stair ascent. Normalized knee adduction moment impulse, as well as the first and second peaks were not different between groups. During stair descent, a similar increase in stance time was found for both osteoarthritis groups.

Significance: The absence of a significant effect of groups on the normalized knee adduction moment during stair negotiation may be explained by a lower ambulatory speed in the obese knee osteoarthritis group, that effectively lowers vertical ground reaction force. Decreasing ambulatory speed may be an effective strategy to lower knee adduction moment during stair negotiation.
\end{abstract}

\section{Introduction}

Osteoarthritis (OA) is a common musculoskeletal disease, characterized by cartilage degradation and subchondral bone deformations that can occur in any joint [1]. Most commonly, OA manifests at the knee joint where it locally causes pain, movement limitations, tenderness, and inflammation [2]. According to the World Health Organization, OA is the fourth leading cause of immobility, with a prevalence of $18 \%$ for women and $9.6 \%$ for men aged above 60 years [3]. Prevalence of knee OA increases with age, especially above 50 years. This agerelated increase in prevalence is further amplified by the female gender [4]. Another important risk factor for knee OA is obesity. Research has shown that obese subjects have almost four times the risk of developing knee OA when compared with non-obese subjects, which may be explained by both an increased knee loading as well as chronic low-grade inflammation $[5,6]$.

In most patients, knee OA is localized at the medial compartment [7]. Medial knee loads may be increased due to lateral laxity and varus alignment [8]. Considering that the medial compartment is not adapted to extreme loads, cartilage may deteriorate when the knee is repeatedly exposed to high joint forces during locomotor activities. Currently, it is believed that particularly high knee adduction moments (KAM) correlates with cartilage loss in the knee [9]. Several studies have already examined the KAM in patients with knee OA during level walking [10-12]. Here, especially the first KAM peak together with the cumulative load, determined from the impulse, are shown to be

\footnotetext{
* Corresponding author.

E-mail address: 1.verlaan@mumc.nl (L. Verlaan).
} 
discriminative for the presence, severity and rate of progression of medial compartment knee OA $[9,13]$. In addition, obesity has been reported to augment KAM during level walking [14]. However, after removal of the direct contributions of body weight, KAM parameters may be different due to obesity-related level walking adaptations to limit knee loading $[14,15]$.

KAM is dependent of the magnitude of the ground reaction force (GRF) and its moment arm relative to the knee joint centre [16]. During level walking, the GRF vector usually passes medially of the knee. While KAM has been thoroughly investigated during level walking, little is known about KAM during stair negotiation [17], during which knee loads are higher compared to level walking [18]. Second, stair climbing is one the first encountered problems in knee OA patients and is often used to evaluate safety of hospital discharge [19].

Although literature on KAM during stair negotiation in knee OA patients is scarce, several factors, including mechanical alignment, lateral trunk lean, toe-out gait, step width, and stair climbing velocity have been suggested to modify KAM during stair negotiation [20-22]. Those movement adaptations should thus be taken into consideration when evaluating KAM. Furthermore, it is important to discriminate between lean and obese knee OA patients [23]. The aim of the current study is therefore to compare normalized KAM during the stance phase of stair negotiation between lean knee OA patients, obese knee OA patients, and healthy controls. As a secondary aim we also sought to explore select characteristics that could impact KAM such as ground reaction force, spatiotemporal parameters and toe-out angle. At last we want to investigate the relationship between normalized KAM during stair negotiation and level walking.

\section{Methods}

\subsection{Study population}

This cross-sectional study included three groups: obese knee OA (body mass index (BMI): $30-40 \mathrm{~kg} \cdot \mathrm{m}^{-2}$ ), lean knee OA (BMI: 20$25 \mathrm{~kg} \cdot \mathrm{m}^{-2}$ ) and healthy controls (BMI: $20-25 \mathrm{~kg} \cdot \mathrm{m}^{-2}$ ). Only women aged between 50 and 65 years were included in study, as knee OA prevalence is highest in this group. The upper aged limit was adopted to prevent inclusion of participants at high risk of having comorbidities (e.g. type II diabetes mellitus, osteoporosis, dementia, cardiovascular disease). OA patients having a Kellgren Lawrence (KL) score between 1 and 3 at the medial tibiofemoral site were included in this study. Recruitment of knee OA patients occurred via the 'Artrose Kliniek' at the Maastricht University Medical Center (MUMC+), The Netherlands. Healthy controls were recruited by the department of Nutrition and Movement Sciences, the department of Physical Therapy (MUMC+), and local physical therapy clinics in Maastricht, The Netherlands.

Exclusion criteria were any inflammatory arthritis, trauma, OA at any other joint in the lower extremities including patellofemoral OA and tibiofemoral OA on the lateral site, anterior cruciate ligament injury, medial and collateral ligament injury, and psychiatric illness according to the Diagnostic and Statistical Manual of Mental Disorders classification criteria for psychiatric illnesses (patients were excluded when diagnoses were present in their medical files). Healthy women were non-obese, did not meet the exclusion criteria, and did not have knee OA according to the American College of Rheumatology classification criteria [24].

All subjects gave informed consent before participating in this study. This study was ethically approved by the METC aZM/UM.

\subsection{Radiographic imaging}

Radiographic imaging was used to evaluate knee cartilage and knee OA status. Presence of knee OA was assessed from X-ray images by the Kellgren-Lawrence knee score [25]. The X-ray images were evaluated double blind by two independent orthopaedic surgeons.
To more accurately assess cartilage health in all study groups, Magnetic Resonance Imaging (MRI) was performed using a 3 T Philips Intera Scanner (Philips Medical Systems, Best, The Netherlands). Cartilage health was evaluated based on the MRI Osteoarthritis Knee Score (MOAKS) [26]. For a more detailed description of imaging procedures, we refer to Verlaan et al [27].

\subsection{Instrumentation}

Motion analysis was performed with an eight camera, three-dimensional (3D) motion capture system (Vicon, MX3, Oxford Metric, United Kingdom) together with Nexus software V1.8. Kinetic data were obtained by one force platform (9281A, Kistler instruments AG, Winethur, Switzerland) which was incorporated in the first step of a twostep staircase. For reliable assessment of point of application of the GRF, the height of the stairs was taken into account. Sixteen reflective markers were placed on the lower extremities according to the Vicon Plug in Gait model in order to use the 3D motion capture system. In the obese knee OA group, however, there were occasional deviations from the model when the abdominal fat depot limited visibility of the markers on the spina iliaca anterior superior. In accordance with the Vicon Plug-in Gait Reference Guide, makers were then placed more dorsal and/or lateral. Correction for these deviations occurred by manually inserting the true distance between the left and right spina iliaca anterior superior into the system.

\subsection{Procedure}

Subjects were asked to ascend and descend the stairs barefooted at a self-selected, comfortable speed. The staircase consisted of one step (height $=20 \mathrm{~cm}$, length $=30 \mathrm{~cm}$; width $=80 \mathrm{~cm}$ ) which contained an embedded force plate, and a platform (height $=20 \mathrm{~cm}$, length $=60 \mathrm{~cm}$, width $=80 \mathrm{~cm}$ ). For safety reasons a railing was added to the staircase (Fig. 1). Only one foot was placed at the step containing the force plate, corresponding to a step-over-step stair negotiation pattern. As a way of standardization, healthy subjects used their dominant leg, while knee OA patients used their affected leg to land on the step containing the force plate. Test trials were allowed for movement familiarization. Measurements were repeated with $10 \mathrm{~s}$ of resting intervals, until at least five successful trials were recorded.

During level walking analysis, subjects were asked to walk barefooted at self-selected walking speed. They were instructed to walk without aiming for the force platform in such a way that the fifth step landed completely on the force platform (i.e. the dominant leg for healthy subjects and the affected leg for OA patients).

\subsection{Data analysis}

Data were processed via a dedicated MATLAB routine. Stair ascent and descent were analysed separately to generate the biomechanical parameters of study. Those parameters included: stance phase duration, toe-out angle, KAM peaks, KAM dip, KAM impulse, and vertical (GRFz) and medio-lateral (GRFx) ground reaction forces. Positive values of the GRFz and GRFx correspond to the vertical and medial directed GRFs respectively. KAM impulse was obtained via numerical integration, using the trapezoid method, of the KAM-stance time curve. The toe-out angle was defined as the angle between the foot vector and sagittal laboratory axis. All parameters were normalized to the stance phase with intervals of $0.5 \%$. Stance phase was defined as the time from heel strike till toe-off, which was based on GRF. Further, joint moments and the vertical ground reaction force were normalized for body mass to remove the direct effects of body mass on KAM parameters.

\subsection{Statistical analysis}

Variables were tested for normality using the Shapiro-Wilk test. 


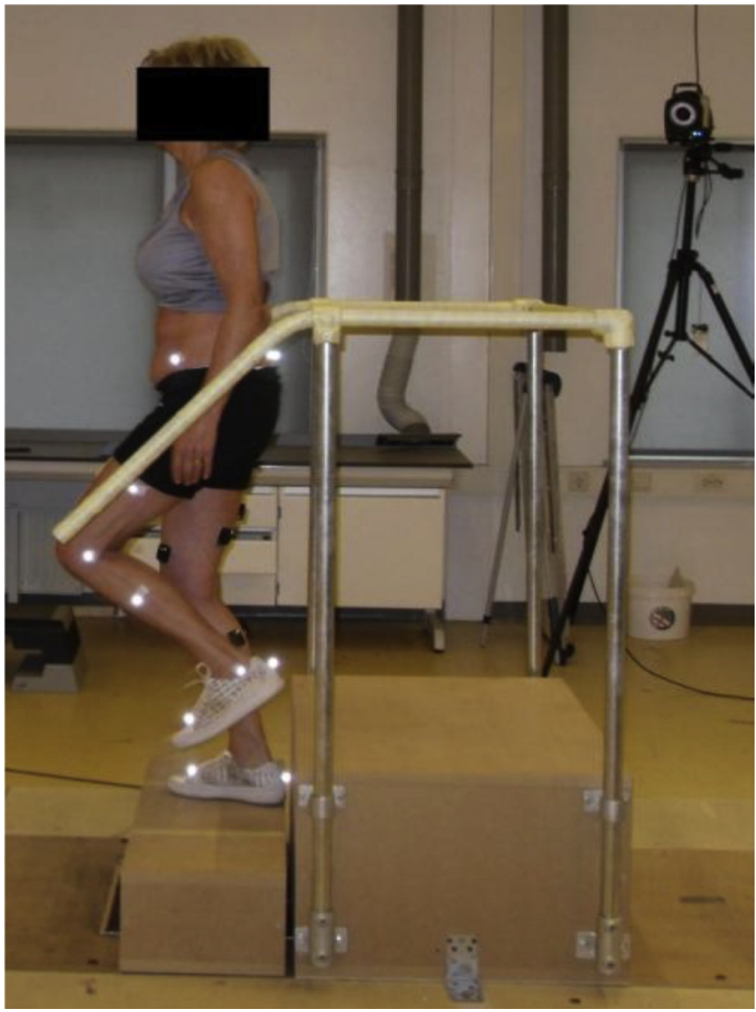

Fig. 1. Staircase used in the experimental set-up. The staircase consisted of one step, containing the embedded force plate and one platform. Note that this picture was obtained during a pilot-session and that no footwear was worn during the actual measurements.

Accordingly, group differences were analysed with one-way ANOVA and non-parametric Kruskal-Wallis tests. Post-hoc analyses were performed using LSD and pairwise comparisons with Bonferroni adjustments respectively. A Chi-Square test was performed to test differences in the distribution of KL scores between groups. Data are presented as mean with standard deviation in brackets. A Pearson's correlation was performed to investigate the relation between toe-out angle and KAM impulse. In addition, the KAM parameters from the current study were correlated with the KAM parameters for the same subjects during level walking at preferred speed. Level of significance was set at $\alpha<0.05$. All statistical analyses were performed with SPSS statistics version 24 (IBM, New York, United States of America).

\section{Results}

\subsection{Subject characteristics}

Fifty subjects were included in this study (Table 1). There were no significant differences in age and height between groups. However, weight and BMI were higher in the obese knee OA group compared to both the controls and lean knee OA group. Radiographic analysis confirmed presence of knee OA in both groups. Distribution of the KL scores was not different between the two knee OA groups for both raters $(P=0.263$ and $P=0.456)$. For the KL scoring, agreement between both orthopaedic surgeons was substantial $(\kappa=0.656)$. Absence of meaningful knee OA in the control group was evidenced by MOAKS scoring, which was significantly lower in the control group, compared to both lean $(P=0.005)$ and obese knee OA groups $(P=0.002)$.

\subsection{Ground reaction force}

In absolute numbers both vertical and medial GRFs were increased
Table 1

Patient characteristics of the study groups, presented as mean (SD).

\begin{tabular}{|c|c|c|c|}
\hline \multirow[t]{2}{*}{ Demographics } & \multicolumn{3}{|l|}{ Group } \\
\hline & $\begin{array}{l}\text { Control } \\
(n=20)\end{array}$ & $\begin{array}{l}\text { Lean knee OA } \\
(\mathrm{n}=14)\end{array}$ & $\begin{array}{l}\text { Obese knee OA } \\
(\mathrm{n}=16)\end{array}$ \\
\hline Age (years) & $58.5(4.7)$ & $60.1(3.4)$ & $58.8(4.6)$ \\
\hline Height (m) & $1.66(0.04)$ & $1.67(0.05)$ & $1.62(0.07)$ \\
\hline Weight (kg) & $62.9(6.2)$ & $66.1(7.3)$ & $86.7(13.1)^{1,2}$ \\
\hline BMI $\left(\mathrm{kg} / \mathrm{m}^{2}\right)$ & $22.7(1.8)$ & $23.8(2.2)$ & $32.7(3.2)^{1,2}$ \\
\hline \multirow[t]{2}{*}{ KL-score $(1 ; 2 ; 3)^{*}$} & - & Rater A: $2 ; 6 ; 6$ & Rater A: $0 ; 8 ; 6$ \\
\hline & - & Rater B: $3 ; 6 ; 5$ & Rater B: $3 ; 4 ; 7$ \\
\hline MOAKS (score/items) & $0.53(0.43)$ & $0.96(0.68)^{1}$ & $1.15(0.60)^{1}$ \\
\hline
\end{tabular}

Note: $\mathrm{BMI}=$ body mass index, $\mathrm{KL}=$ Kellgren Lawrence, MOAKS $=$ MRI osteoarthritis knee score, $\mathrm{OA}=$ osteoarthritis. $1=$ significantly different from control, 2 = significantly different from lean knee OA. * = for two obese knee OA patients no X-ray has been obtained, however, presence of OA was here confirmed by MRI.

in obese knee OA patients $(P<0.050)$ (Table 2). After normalization for body mass, the first peak of the GRFz was reduced in obese knee OA patients compared to healthy controls and lean knee OA patients during stair ascent $(P<0.001)$. During stair descent, only obese knee OA patients showed a lower first peak GRFz compared to healthy controls $(P=0.005)$. The GRFx was only increased in the obese knee OA group during stair ascent, when compared with healthy controls and lean OA patients. During stair descent, the dip of the GRFx during mid-stance was higher in the obese knee OA group compared to both the controls and lean knee OA group.

\section{3. $K A M$ during stair negotiation}

Before normalization, KAM parameters were increased in obese knee OA patients during both stair ascent and descent $(P<0.050)$ (Table 2). However, after normalization obese knee OA patients showed a more flattened KAM curve during both stair ascent and stair descent (Fig. 2). During stair ascent, this pattern in obese knee OA patients was characterized by an increased KAM at the dip compared to both healthy controls $(P=0.003)$ and lean knee OA patients $(P=0.038)$. Both obese $(P<0.001)$ and lean knee OA patients $(P=0.019)$ showed a similar increase of the KAM dip during stair descent, when compared to healthy controls. After normalization, KAM impulse, first KAM peak, and the second KAM peak were not different between groups during stair ascent $(P>0.050)$. Despite high between-subject variability (Fig. 2), KAM impulse was found $45 \%$ higher in the obese knee OA group during stair descent, when compared to healthy controls $(P=0.012)$.

\subsection{Spatiotemporal parameters}

During stair ascent obese knee OA patients showed a $15.7 \%$ longer stance phase than healthy controls $(P<0.001)$ and $8.4 \%$ longer stance phase compared to lean knee OA patients $(P=0.033)$. Both lean and obese knee OA patients also showed increased stance times during stair descent, when compared to healthy controls. For the obese knee OA group this was an increase of $15.7 \%(P=0.001)$, whereas this was $10.7 \%$ for the lean knee OA group $(P=0.019$ ) (Table 2$)$.

\subsection{Relation between toe-out gait and KAM}

There were no differences in toe-out gait during stair negotiation between the different groups. During stair descent, however, for all subjects, toe-out angle correlated with KAM impulse $(r=-0.391$; $P=0.005$ ). Overall, a $1^{\circ}$ increase of toe-out gait was related with a decrease in KAM impulse of $5.3 \times 10^{-3} \mathrm{Nm} * \mathrm{~s} / \mathrm{kg}$. Within groups, however, foot progression angle did not correlate with KAM impulse. 
Table 2

Biomechanical parameters during stair negotiation. Data are presented as mean (SD).

\begin{tabular}{|c|c|c|c|c|c|c|}
\hline \multirow[b]{2}{*}{ Biomechanical parameter } & \multicolumn{3}{|l|}{ Stair Ascent } & \multicolumn{3}{|l|}{ Stair Descent } \\
\hline & Control $(n=20)$ & Lean knee OA $(n=14)$ & Obese knee OA $(n=16)$ & Control $(\mathrm{n}=20)$ & Lean knee OA $(n=14)$ & Obese knee OA $(n=16)$ \\
\hline Stance phase duration (s) & $0.89(0.10)$ & $0.95(0.09)$ & $1.03(0.11)^{1,2}$ & $0.75(0.08)$ & $0.83(0.07)^{1}$ & $0.87(0.13)^{1}$ \\
\hline Toe-out angle $\left({ }^{\circ}\right)$ & $6.5(9.3)$ & $0.7(9.7)$ & $3.6(11.6)$ & $16.1(6.5)$ & $9.9(9.3)$ & $15.2(10.3)$ \\
\hline \multicolumn{7}{|l|}{$K A M(N m)$} \\
\hline 1 st peak & $46.3(9.6)$ & $40.6(16.7)$ & $51.2(11.4)$ & $32.5(10.2)$ & $35.2(7.0)$ & $46.3(18.5)^{1,2}$ \\
\hline Dip & $7.6(5.9)$ & $10.0(8.2)$ & $20.8(10.3)^{1,2}$ & $9.0(4.0)$ & $15.1(6.0)^{1}$ & $25.9(11.8)^{1,2}$ \\
\hline 2nd peak & $17.3(8.6)$ & $19.7(13.5)$ & $33.0(15.4)^{1,2}$ & $38.8(14.1)$ & $41.9(15.5)$ & $56.4(23.0)^{1,2}$ \\
\hline KAM impulse (Nm*s) & $16.1(5.3)$ & $17.0(8.1)$ & $28.3(8.8)^{1,2}$ & $13.8(4.7)$ & $19.1(5.9)$ & $27.6(12.5)^{1,2}$ \\
\hline \multicolumn{7}{|l|}{$\mathrm{KAM}(\mathrm{Nm} / \mathrm{kg})$} \\
\hline 1 st peak & $0.74(0.16)$ & $0.61(0.24)$ & $0.60(0.17)$ & $0.52(0.18)$ & $0.54(0.12)$ & $0.56(0.22)$ \\
\hline Dip & $0.12(0.09)$ & $0.15(0.12)$ & $0.24(0.11)^{1,2}$ & $0.16(0.07)$ & $0.23(0.09)^{1}$ & $0.29(0.14)^{1}$ \\
\hline 2nd peak & $0.28(0.14)$ & $0.30(0.19)$ & $0.39(0.19)$ & $0.62(0.23)$ & $0.64(0.24)$ & $0.66(0.30)$ \\
\hline KAM impulse $(\mathrm{Nm} * \mathrm{~s} / \mathrm{kg})$ & $0.26(0.08)$ & $0.26(0.12)$ & $0.31(0.11)$ & $0.22(0.08)$ & $0.29(0.10)$ & $0.32(0.15)^{1}$ \\
\hline \multicolumn{7}{|l|}{ Medial GRF $(N)$} \\
\hline $1^{\text {st }}$ peak & $24.5(10.0)$ & $24.7(7.56)$ & $39.2(11.2)^{1,2}$ & $40.8(10.4)$ & $43.9(17.2)$ & $62.2(15.4)^{1,2}$ \\
\hline Dip & $3.22(4.9)$ & $5.56(5.6)$ & $11.8(9.2)^{1,2}$ & $5.1(5.7)$ & $7.8(6.9)$ & $19.8(12.5)^{1,2}$ \\
\hline $2^{\text {nd }}$ peak & $20.7(8.33)$ & $20.0(8.9)$ & $35.2(14.8)^{1,2}$ & $25.7(5.6)$ & $28.2(8.9)$ & $43.0(15.3)^{1}$ \\
\hline \multicolumn{7}{|l|}{ Medial GRF (N/kg) } \\
\hline $1^{\text {st }}$ peak & $0.39(0.15)$ & $0.38(0.11)$ & $0.45(0.11)$ & $0.65(0.17)$ & $0.66(0.22)$ & $0.71(0.15)$ \\
\hline Dip & $0.05(0.08)$ & $0.08(0.09)$ & $0.13(0.10)^{1}$ & $0.08(0.09)$ & $0.12(0.10)$ & $0.22(0.12)^{1,2}$ \\
\hline $2^{\text {nd }}$ peak & $0.33(0.13)$ & $0.30(0.13)$ & $0.40(0.15)$ & $0.41(0.09)$ & $0.43(0.13)$ & $0.49(0.13)$ \\
\hline \multicolumn{7}{|l|}{ Vertical GRF (N) } \\
\hline $1^{\text {st }}$ peak & $625.5(65.8)$ & $675.4(83.6)$ & $836.9(119.9)^{1,2}$ & $951.7(104.0)$ & $942.4(169.8)$ & $1171.1(184.4)^{1,2}$ \\
\hline Dip & $432.1(63.8)$ & $497.9(61.1)^{1}$ & $654.0(114.1)^{1,2}$ & $456.8(65.5)$ & $510.9(59.0)$ & $672.5(126.9)^{1,2}$ \\
\hline $2^{\text {nd }}$ peak & $773.8(85.1)$ & $759.2(82.0)$ & $976.2(146.0)^{1,2}$ & $583.4(63.3)$ & $643.8(68.1)$ & $825.4(125.9)^{1,2}$ \\
\hline \multicolumn{7}{|l|}{ Vertical GRF (BW) } \\
\hline $1^{\text {st }}$ peak & $1.06(0.04)$ & $1.04(0.04)$ & $0.99(0.04)^{1,2}$ & $1.53(0.13)$ & $1.45(0.17)$ & $1.38(0.16)^{1}$ \\
\hline Dip & $0.70(0.07)$ & $0.77(0.05)^{1}$ & $0.77(0.06)^{1}$ & $0.74(0.06)$ & $0.79(0.04)^{1}$ & $0.79(0.09)^{1}$ \\
\hline $2^{\text {nd }}$ peak & $1.26(0.08)$ & $1.17(0.07)^{1}$ & $1.15(0.12)^{1}$ & $0.95(0.06)$ & $1.00(0.05)$ & $0.97(0.07)$ \\
\hline
\end{tabular}

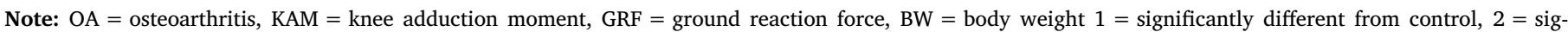
nificantly different from lean knee OA.

\subsection{Correlations between level walking and stair negotiation}

Irrespective of the study group, KAM patterns were comparable between level walking and stair negotiation (Fig. 2). Especially the dip $(r=0.846 ; P<0.001)$ and the second peak $(r=0.770 ; P<0.001)$ of KAM during stair ascent showed high correlations with KAM during level walking. First peak KAM during stair ascent correlated with the first peak during level walking $(r=0.310 ; P=0.041)$. When considering stair descent, the dip of KAM correlated with KAM dip during level walking $(r=0.618 ; P<0.001)$. Additionally, the first peak correlated with the first peak during level walking $(r=0.419$; $P=0.004)$.

\section{Discussion}

In the current study, obese knee OA subjects showed a more flattened KAM curve during stair ascent. During stair descent, both OA groups showed a similar increase in KAM dip at mid-stance, compared to healthy controls. This corresponds with increases in medial and vertical GRF during mid-stance in the obese knee OA group, which may relate with increased stance times of obese knee OA patients during both stair ascent and stair descent. Lastly, KAM parameters were related when compared between level walking and stair negotiation, indicating task similarities.

Previous studies on stair negotiation in knee OA patients showed no significant effect of knee OA on normalized KAM peaks in obese knee OA patients during stair climbing [11,22], as also found in this study. Without normalization, KAM parameters were significantly increased in obese knee OA patients, which is caused by the direct contribution of body mass to KAM. The absence of statistical significance of the normalized KAM peaks does however not indicate that KAM is not an important parameter for stair negotiation [17]. Flattened KAM curves, characterized by a less pronounced dip at mid-stance, have previously been recognized in obese and severe knee OA patients during level walking [28]. Therefore, cumulative load (i.e. KAM impulse) was proposed as more accurate indicator for differences between the study groups, as the impulse is not restricted to a certain timepoint $[29,30]$. No significant differences in normalized KAM impulse were found between the different groups during stair ascent, whereas obese knee OA patients have an increased impulse during stair descent. We suggest that the absence of statistical significance for the KAM parameters may be explained by compensatory movement strategies, such as ambulatory velocity. Increases in stance time were found in obese knee OA patients, implying a decrease in ambulatory velocity. A decrease in vertical acceleration lowers the vertical GRF and thus may lead to a reduction in KAM [31]. Indeed, both peaks of the vertical GRF were decreased during stair ascent in both the obese and lean knee OA group, when compared with healthy controls. Furthermore, a decrease in ambulatory velocity is accompanied by an increase in vertical GRF at mid stance. Besides the KAM peaks and dip, ambulatory velocity directly affects KAM impulse as this is the integral of KAM over the stance duration. Despite the increased stance time in the obese knee OA group, KAM impulse did not differ between groups during stair ascent.

Reasons for this may include the high observed heterogeneity in KAM in both obese and lean knee OA patients (Fig. 2). This betweensubject variability may indicate differential use of compensatory mechanisms that could reduce KAM and thus underlie the non-significance found during stair ascent. For example, the present study showed that only obese knee OA patients tend to prolong their stance phase during stair ascent, whereas both obese and lean OA have prolonged stance phases during stair descent. In addition, a significant correlation between toe-out gait and KAM impulse was found during stair descent. By increasing the toe-out angle the knee joint axis is rotated externally. As a result, the GRF will pass more posterior and less medial of the knee joint centre, which leads to conversion of KAM into a knee flexion moment [28]. Increased toe-out gait also causes a shift of the centre of 


\section{A)}
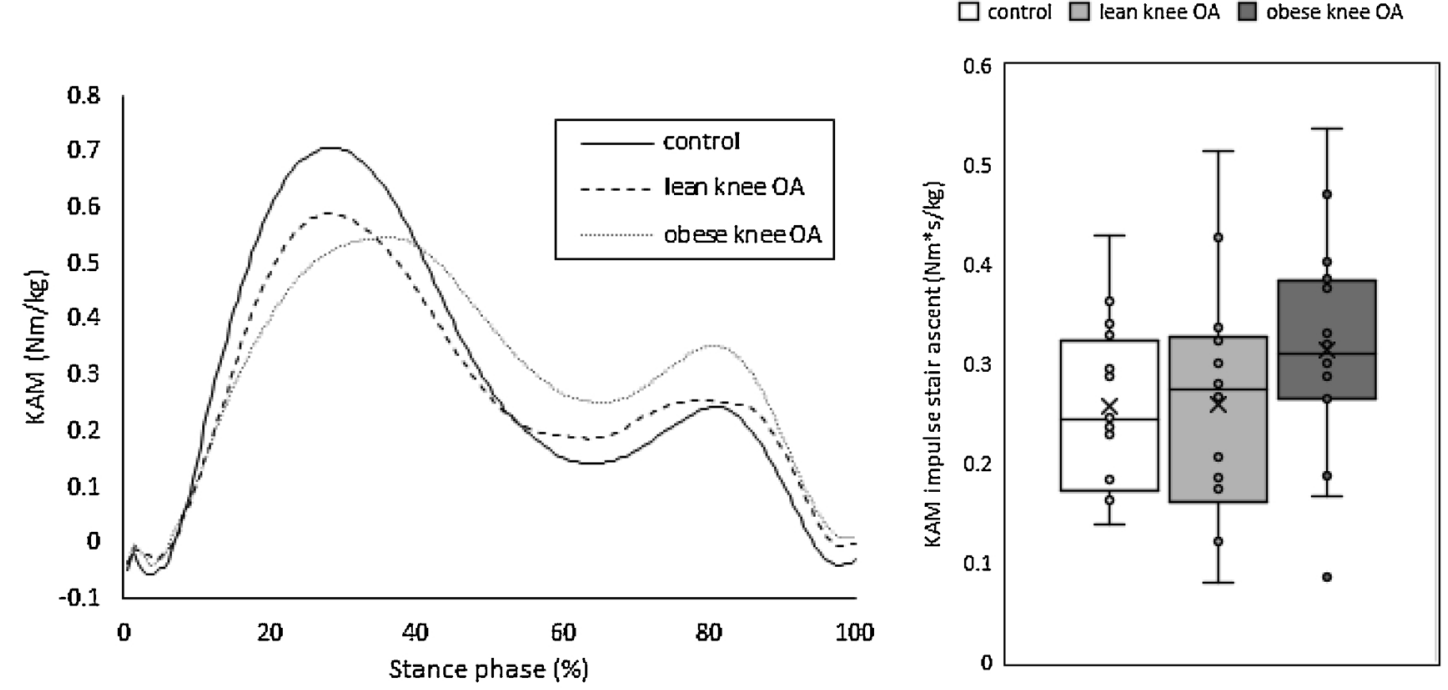

$\square$ control $\square$ lean knee OA $\square$ obese knee OA

B)

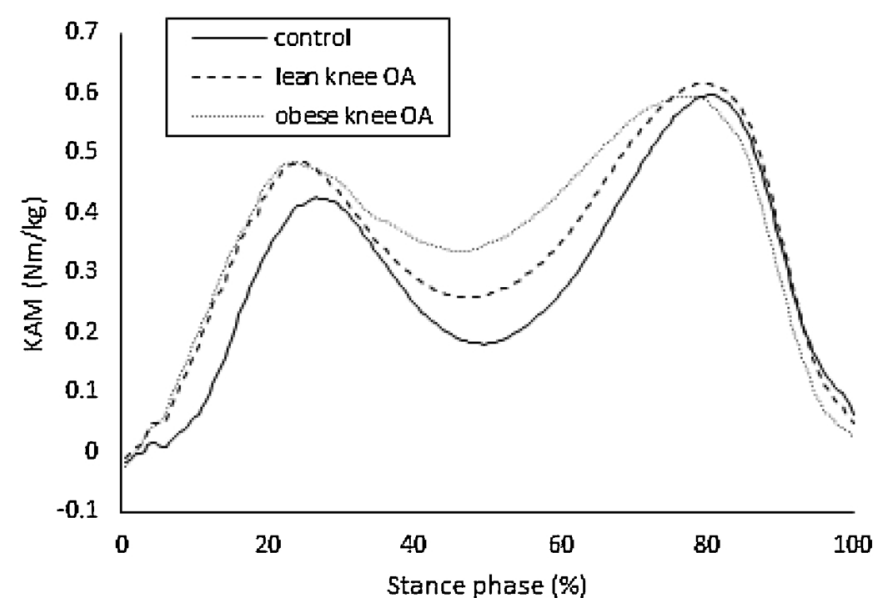



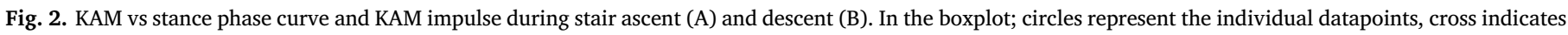
the group mean value. Upper and lower quartiles are indicated by the whiskers, the boxes represent the interquartile range.

pressure during late stance, resulting in a GRF vector with a lower lever arm which lowers the second KAM peak [21]. Although we found a correlation between toe-out gait and KAM impulse, there were no indications for differential use of toe-out gait between groups. Therefore, we believe that, besides lowering ambulatory speed, increased toe-out gait during stair negotiation may further benefit obese knee OA patients.

Furthermore, similarities in KAM patterns were found between level walking and stair negotiation (Fig. 3). This suggests that movement strategies are not altered between different activities of daily living. In other words, those who successfully reduce KAM during level walking, seem to be able to adequately reduce KAM during stair negotiation as well. Clinically, this would imply that focus could be intensified on reducing KAM for only one activity of daily living and the reductions in the other may follow. Furthermore, it implies that there may be similarities in biomechanical models underlying level walking and stair negotiation.

The present study design allowed investigation to compare KAM between knee OA in absence and presence of obesity. However, there were some limitations. Although we tried to optimize marker placement, markers did not exactly coincide with the anatomical landmarks, which could be a small source of error. Furthermore, soft tissue artifacts are expected to be more prominent in the obese population, introducing additional variability. However, given the fact that variability of KAM parameters is comparable between the lean and obese knee OA group, we believe the contribution of soft tissue artifacts is not disparate between groups. Nevertheless, the overall high variability in KAM parameters might also be attributed to inaccuracies of the Plug-in-Gait model in the frontal plane. The lack of a fourth control group, being weight matched obese control subjects, limits our interpretations of the individual effect of obesity on KAM. Due to the fact trunk biomechanics were not investigated, effects of lateral trunk lean on KAM cannot be excluded. Future studies should therefore investigate lateral trunk lean in relation to KAM in lean and obese knee OA patients, with the addition of a fourth control group.

\section{Conclusions}

The current study showed that, after removing the direct effects of body mass, there are no significant differences between the study groups in the first and second KAM peaks during stair negotiation. Possible reasons for this may include increased stance times for obese knee OA patients that lower vertical GRF and thus KAM. As a side effect, KAM dip at mid-stance was increased in obese knee OA patients. 
$1^{\text {st }}$ peak KAM
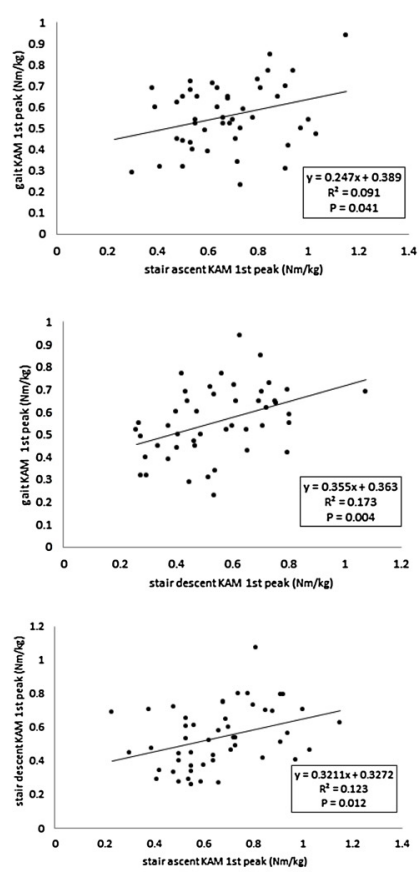

KAM dip
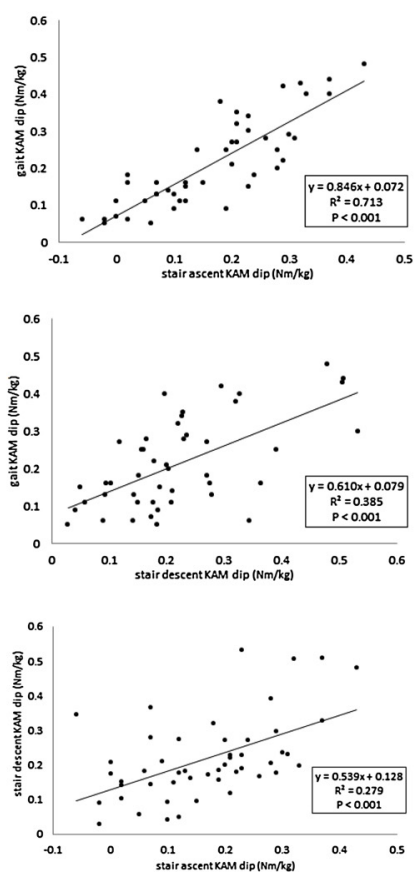

$2^{\text {nd }}$ peak $K A M$
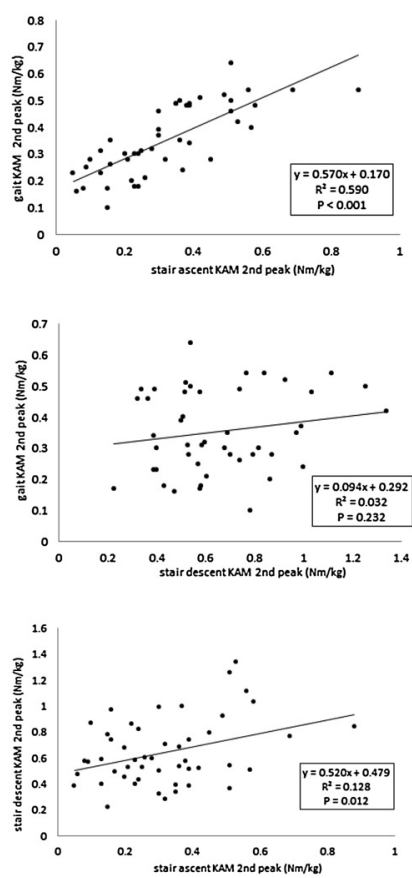

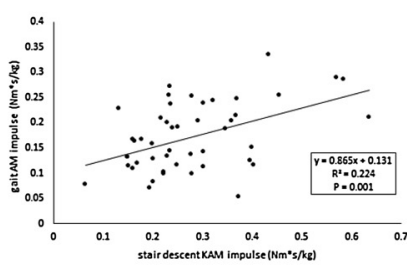

KAM impulse
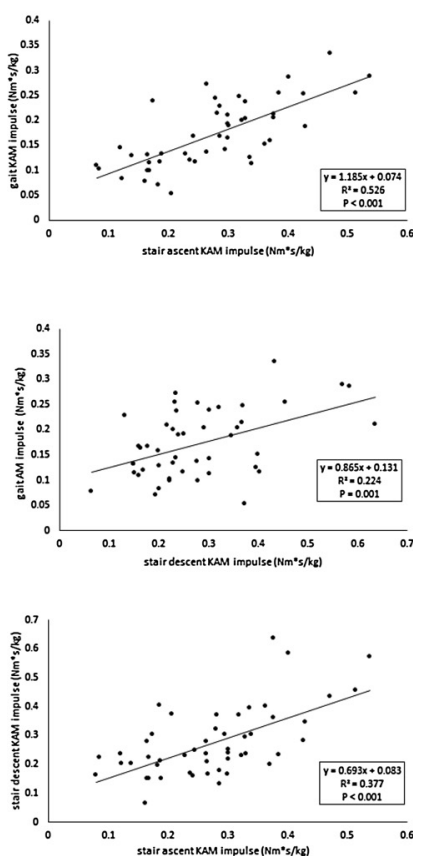

Fig. 3. Correlations of KAM parameters between gait and stair negotiation for all subjects.

Increased toe-out gait may also lower KAM. However, there were no indications for differential use of this compensatory strategy between study groups. Furthermore, significant correlations between KAM parameters were found during stair negotiation and level walking.

\section{Funding}

This research did not receive any specific grant from funding agencies in the public, commercial, or not-for-profit sectors.

\section{CRediT authorship contribution statement}

L. Verlaan: Conceptualization, Methodology, Validation, Investigation, Data curation, Writing - original draft. R.J. Boekesteijn: Software, Formal analysis, Data curation, Writing - original draft. P.W. Oomen: Investigation. W.Y. Liu: Investigation. M.J.M. Peters: Software, Validation. P.J. Emans: Resources, Supervision. L.W. van Rhijn: Resources, Supervision. K. Meijer: Conceptualization, Methodology, Resources, Supervision.

\section{Declaration of Competing Interest}

None of the authors have competing interests to declare. This research did not receive any specific grant from funding agencies in the public, commercial, or not-for-profit sectors.

\section{References}

[1] K.D. Allen, Y.M. Golightly, Epidemiology of osteoarthritis: state of the evidence, Curr. Opin. Rheumatol. 27 (3) (2015) 276-283.

[2] K.M. Jordan, N.K. Arden, M. Doherty, B. Bannwarth, J.W. Bijlsma, P. Dieppe, et al., EULAR Recommendations 2003: an evidence based approach to the management of knee osteoarthritis: report of a Task Force of the Standing Committee for International Clinical Studies including Therapeutic Trials (ESCISIT), Ann. Rheum. Dis. 62 (12) (2003) 1145-1155.

[3] A.D. Woolf, B. Pfleger, Burden of major musculoskeletal conditions, Bull. World Health Organ. 81 (9) (2003) 646-656.

[4] N. Arden, M.C. Nevitt, Osteoarthritis: epidemiology, Best Pract. Res. Clin. Rheumatol. 20 (1) (2006) 3-25.

[5] D. Coggon, I. Reading, P. Croft, M. McLaren, D. Barrett, C. Cooper, Knee osteoarthritis and obesity, Int. J. Obes. Relat. Metab. Disord. 25 (5) (2001) 622-627.

[6] W.H. Robinson, C.M. Lepus, Q. Wang, H. Raghu, R. Mao, T.M. Lindstrom, et al., Low-grade inflammation as a key mediator of the pathogenesis of osteoarthritis, Nat. Rev. Rheumatol. 12 (10) (2016) 580-592.

[7] F. Cicuttini, A. Wluka, J. Hankin, Y. Wang, Longitudinal study of the relationship between knee angle and tibiofemoral cartilage volume in subjects with knee osteoarthritis, Rheumatology (Oxford) 43 (3) (2004) 321-324.

[8] L. Sharma, J. Song, D.T. Felson, S. Cahue, E. Shamiyeh, D.D. Dunlop, The role of knee alignment in disease progression and functional decline in knee osteoarthritis, JAMA 286 (2) (2001) 188-195.

[9] L. Sharma, D.E. Hurwitz, E.J. Thonar, J.A. Sum, M.E. Lenz, D.D. Dunlop, et al., Knee adduction moment, serum hyaluronan level, and disease severity in medial tibiofemoral osteoarthritis, Arthritis Rheum. 41 (7) (1998) 1233-1240.

[10] A.J. Baliunas, D.E. Hurwitz, A.B. Ryals, A. Karrar, J.P. Case, J.A. Block, et al, Increased knee joint loads during walking are present in subjects with knee osteoarthritis, Osteoarthr. Cartil. 10 (7) (2002) 573-579.

[11] K.R. Kaufman, C. Hughes, B.F. Morrey, M. Morrey, K.N. An, Gait characteristics of patients with knee osteoarthritis, J. Biomech. 34 (7) (2001) 907-915.

[12] S.C. Landry, K.A. McKean, C.L. Hubley-Kozey, W.D. Stanish, K.J. Deluzio, Knee biomechanics of moderate OA patients measured during gait at a self-selected and fast walking speed, J. Biomech. 40 (8) (2007) 1754-1761.

[13] L.E. Thorp, D.R. Sumner, M.A. Wimmer, J.A. Block, Relationship between pain and medial knee joint loading in mild radiographic knee osteoarthritis, Arthritis Rheum. 57 (7) (2007) 1254-1260.

[14] N.A. Segal, H.J. Yack, P. Khole, Weight, rather than obesity distribution, explains peak external knee adduction moment during level gait, Am. J. Phys. Med. Rehabil. 88 (3) (2009) 180-246.

[15] G.T. Harding, C.L. Hubley-Kozey, M.J. Dunbar, W.D. Stanish, J.L. Astephen Wilson, Body mass index affects knee joint mechanics during gait differently with and without moderate knee osteoarthritis, Osteoarthr. Cartil. 20 (11) (2012) 1234-1242.

[16] N. Foroughi, R. Smith, B. Vanwanseele, The association of external knee adduction moment with biomechanical variables in osteoarthritis: a systematic review, Knee 16 (5) (2009) 303-309.

[17] H. Iijima, K. Shimoura, T. Aoyama, M. Takahashi, Biomechanical characteristics of stair ambulation in patients with knee OA: a systematic review with meta-analysis toward a better definition of clinical hallmarks, Gait Posture 62 (2018) 191-201.

[18] I. Kutzner, B. Heinlein, F. Graichen, A. Bender, A. Rohlmann, A. Halder, et al., Loading of the knee joint during activities of daily living measured in vivo in five subjects, J. Biomech. 43 (11) (2010) 2164-2173.

[19] P.A. Costigan, K.J. Deluzio, U.P. Wyss, Knee and hip kinetics during normal stair climbing, Gait Posture 16 (1) (2002) 31-37.

[20] J. Lewis, G. Freisinger, X. Pan, R. Siston, L. Schmitt, A. Chaudhari, Changes in lower extremity peak angles, moments and muscle activations during stair climbing at different speeds, J. Electromyogr. Kinesiol. 25 (6) (2015) 982-989.

[21] M. Guo, M.J. Axe, K. Manal, The influence of foot progression angle on the knee adduction moment during walking and stair climbing in pain free individuals with knee osteoarthritis, Gait Posture 26 (3) (2007) 436-441. 
[22] M.R. Paquette, G. Klipple, S. Zhang, Greater step widths reduce internal knee abduction moments in medial compartment knee osteoarthritis patients during stair ascent, J. Appl. Biomech. 31 (4) (2015) 229-236.

[23] R.C. Browning, R. Kram, Effects of obesity on the biomechanics of walking at different speeds, Med. Sci. Sports Exerc. 39 (9) (2007) 1632-1641.

[24] R. Altman, E. Asch, D. Bloch, G. Bole, D. Borenstein, K. Brandt, et al., Development of criteria for the classification and reporting of osteoarthritis. Classification of osteoarthritis of the knee. Diagnostic and Therapeutic Criteria Committee of the American Rheumatism Association, Arthritis Rheum. 29 (8) (1986) 1039-1049.

[25] J.H. Kellgren, J.S. Lawrence, Radiological assessment of osteo-arthrosis, Ann. Rheum. Dis. 16 (4) (1957) 494-502.

[26] D.J. Hunter, A. Guermazi, G.H. Lo, A.J. Grainger, P.G. Conaghan, R.M. Boudreau, et al., Evolution of semi-quantitative whole joint assessment of knee OA: MOAKS (MRI Osteoarthritis Knee Score), Osteoarthr. Cartil. 19 (8) (2011) 990-1002.
[27] L. Verlaan, R.J. Boekesteijn, P.W. Oomen, W.-Y. Liu, M.J.M. Peters, M.A. Witlox, et al., Biomechanical alterations during sit-to-stand transfer are caused by a synergy between knee osteoarthritis and obesity, Biomed Res. Int. 2018 (2018) 7.

[28] N.D. Reeves, F.L. Bowling, Conservative biomechanical strategies for knee osteoarthritis, Nat. Rev. Rheumatol. 7 (2) (2011) 113-122.

[29] D.J. Rutherford, C.L. Hubley-Kozey, K.J. Deluzio, W.D. Stanish, M. Dunbar, Foot progression angle and the knee adduction moment: a cross-sectional investigation in knee osteoarthritis, Osteoarthr. Cartil. 16 (8) (2008) 883-889.

[30] L.E. Thorp, D.R. Sumner, J.A. Block, K.C. Moisio, S. Shott, M.A. Wimmer, Knee joint loading differs in individuals with mild compared with moderate medial knee osteoarthritis, Arthritis Rheum. 54 (12) (2006) 3842-3849.

[31] A. Protopapadaki, W.I. Drechsler, M.C. Cramp, F.J. Coutts, O.M. Scott, Hip, knee, ankle kinematics and kinetics during stair ascent and descent in healthy young individuals, Clin. Biomech. (Bristol, Avon) 22 (2) (2007) 203-210. 